\title{
Contrast attenuation characteristics of iris clipped intraocular lens implants in situ*
}

\author{
ROBERT F HESS, ' GEORGE C WOO, 'AND PAUL D WHITE ${ }^{3}$ \\ From the 'Physiological Laboratory, University of Cambridge; the ${ }^{2} S$ chool of Optometry, University of \\ Waterloo, Canada; and the ${ }^{3}$ Kitchener Waterloo Hospital, Kitchener, Canada
}

SUMMARY In this study contrast thresholds were monitored over a wide range of spatial frequencies for patients with iris clipped implants or Fyodorov and Binkhorst types. These results were compared with those of an age matched normal group and attenuation characteristics derived for the implants and media environment alone. These results show that lenticular implants of this type can produce large reductions in contrast with a wide variety of transfer functions, for example, low pass, band reject, and band pass. These findings highlight the potential inadequacies of the assessment of the visual capabilities of these devices by acuity measures alone. We suggest that a more realistic and abbreviated approach should be based upon supplementing acuity assessment with monitoring of threshold sensitivity for a low, medium, and high spatial frequency sinewave grating.

Intraocular lens implants have been adopted as one of the treatments for aphakics since the technique was first employed by Ridley in 1949.' Binkhorst introduced his iris clipped lens in $1957 .{ }^{2}$ Many modifications followed his design, which include the Fyodorov sputnik and Worst iris clipped lenses. The most prevalent intraocular lens (IOL) through the 1960 s and up to 1978 was the iris clipped lens. At present iris clipped lenses are seldom used. The trend is towards using either anterior chamber or posterior chamber intraocular lenses. The United States Federal Drug Administration report ${ }^{3}$ on IOLs showed that over one million of them have been implanted.

Kayes $^{4}$ reported that $85 \%$ of his first 100 consecutive medallion lens implants had visual acuities better than $6 / 12$ five to seven years after surgery. Hurite and Lempert ${ }^{5}$ stated that $93 \%$ of the 1442 cases of extracapsular cataract extraction with IOLs had 6/12 and better visual acuity. Statistics on visual acuities with IOLs presented by Stark matched those in the previous two studies. ${ }^{3}$

Reduced distance Snellen acuities through IOLs have been attributed to a variety of factors. They

*Presented in part at the mecting of the Association of Rescarch in Vision and Ophthalmology in Sarasota, Florida, in May 1981.

Correspondence to Dr R F Hess, Kenneth Craig Laboratory, Department of Physiology, Cambridge CB2 3EG. include: increasing patient age, surgical problems, postoperative complication, and adverse reactions. ${ }^{3}$ Invariably vision has been assessed only with the use of conventional Snellen acuity charts. Contrast sensitivity measurement has been very successful in the evaluation of normal and abnormal visual function..$^{6-8}$ Its approach extends the present Snellen letter acuity assessment, which takes account only of high contrast acuity objects. The new approach to measurement of contrast sensitivity for object sizes within the resolution limit offers not only a more complete description of different types of visual loss but also a more sensitive one. It is hoped that these two factors will contribute to a method that correlates with perception. It is with this hope in mind that we have applied this approach to the assessment of the visual function of IOL implants. We find that some iris clipped IOL implants produce a wide variety of contrast attenuation that cannot be appreciated on the basis of acuity assessment alone.

\section{Materials and methods}

Vertical sinewave gratings of variable frequency and contrast were generated on the screen on an oscilloscope. The screen's mean luminance was set to 18 $\mathrm{cd} / \mathrm{m}^{2}$, and contrast was modulated about this luminance and adjusted by the patient with an 80 
position switched logarithmic attenuator. Viewing distances varied from $570 \mathrm{~cm}$ for high spatial frequencies to $114 \mathrm{~cm}$ for low spatial frequencies. The method of adjustment was used to set thresholds. The patient's task was to respond when he first discerned non-uniformity of a field presented to him. Five thresholds were obtained for each of the predetermined grating frequencies. These patients were seen between three months and two years after surgery and refracted carefully for residual refractive errors. The IOL implants were inserted by one of us (P.D.W.) and were of either the Fyodorov or Binkhorst iris clipped type. Both Snellen acuities and contrast sensitivity functions were obtained for each subject (10 eyes). Visual acuities were determined with a $\log$ MAR chart ${ }^{4}$ and converted into their Snellen equivalents (see Table 1).

\section{Results}

The contrast sensitivity assessment of 10 normal eyes is displayed in Fig. 1. The $95 \%$ confidence limit for normal vision extends to cover approximately a factor of two in contrast sensitivity at each spatial frequency. These subjects were age matched to our patient sample and were also similarly inexperienced in psychophysical tests. These results showed that, even though any one subject's result could be repeated to an accuracy of $\pm 2 \mathrm{~dB}$ of contrast sensitivity $( \pm 25 \%)$, there was a genuine range of absolute variability between eyes.

Since two of our patients were uniocularly pseudo-

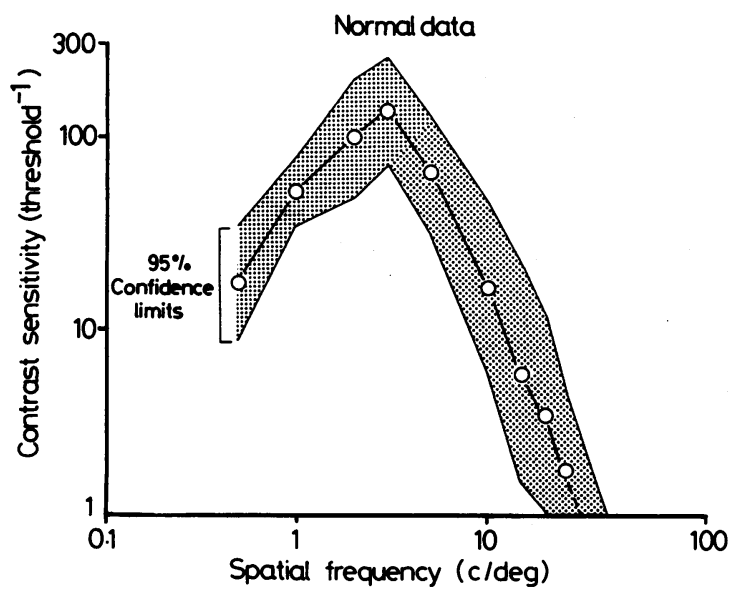

Fig. 1 The relationship between contrast sensitivity (threshold ${ }^{\prime}$ ) is plotted against spatial frequency (cycles of the grating per visual degree) for grating stimuli with a sinusoidal luminance profile. The data for 10 normal subjects, whose ages are matched to our patient population, are shown. Open symbols show the mean results. The stippled area represents $\pm 95 \%$ confidence limits $( \pm 2 S D)$. phakic with a normal fellow eye, we had the opportunity to compare their normal eye's response with that of our normal, matched control group. In Fig. 2 the $95 \%$ confidence limits of our normal population, shown as a shaded area, is compared with the results for normal eyes (unfilled symbols) and eyes with intraocular implants. The results for the two normal eyes of our patient group compared well with the mean of our normal population.

The contrast sensitivity results through IOL lenses were made between three months and two years after implantation to minimise the effects of postsurgical trauma or cystoid macular oedema known to occur sometimes after surgery. An ophthalmological assessment of each patient made by one of us (P.D.W.) at the time of testing indicated no obvious corneal oedema or cystoid macular oedema except in patient six, who showed postsurgical cystoid macular oedema.

Seven of our patients showed large and highly significant losses of contrast sensitivity. Unexpectedly the form of the loss in sensitivity was not of a unitary type. In patients 2 and 5 the contrast sensitivity loss was minimal at low spatial frequencies and increased with increasing spatial frequency. In patients 3,4 , and 8 the contrast sensitivity loss was restricted mainly to mid spatial frequencies. Sensitivity was close to normal for very high $(40 \mathrm{c} / \mathrm{deg})$ and very low $(0.5 \mathrm{c} / \mathrm{deg})$ spatial frequencies. In the case of patient 6 , who suffered postsurgical complications, contrast sensitivity losses were seen for all spatial frequencies, but intermediate spatial frequencies were relatively less affected.

The selective loss of contrast sensitivity for intermediate spatial frequencies, which represented the largest response category, could not be detected by the measurement of grating acuity alone. In Fig. 3 the loss of contrast sensitivity at $3 \mathrm{c} / \mathrm{deg}$ is compared with loss of grating acuity. It is obvious (as it is from the results in Fig. 2) that, although there is good correlation between these two measures, there is a significant non-zero $y$ intercept. This means that grating acuity began to change only after thresholds for mid spatial frequencies were raised by a factor of approximately three. For contrast losses of less than a factor of three grating acuity was a poor predictor of contrast transmission at intermediate spatial frequencies. Although only two Binkhorst implants were tested, there seemed to be no obvious difference between the Fyodorov and Binkhorst types compared with the great variety of responses with any one implant type. Table 1 gives the Snellen acuity of all patients. It is clear from a comparison of these acuities with the contrast loss in Fig. 2 that Snellen acuity, like grating acuity, was also a poor predictor of the overall visual quality of the implant. 

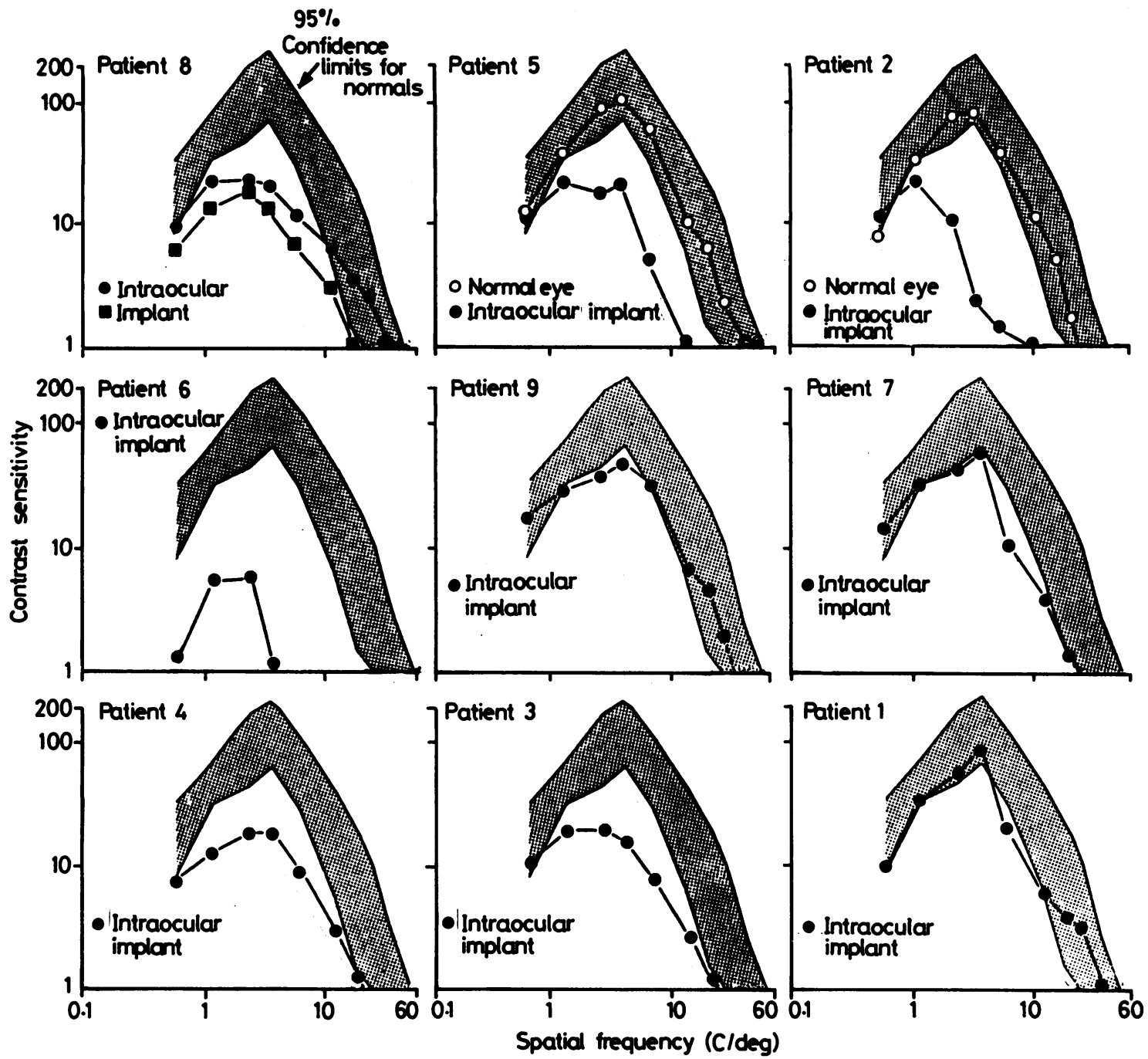

Fig. 2 Contrast sensitivity functions are shown for the normal $(\bigcirc)$ eyes and eyes with intraocular implants $(0)$. These are compared with the $95 \%$ confidence limits for age matched normals. The losses in contrast sensitivity for the intraocular implant are large and vary considerably from patient to patient.

The contrast filtering properties of the iris clipped IOL implant and media environment could be approximately calculated from the present results by taking the ratio of sensitivity for the normal intact eye and the lens implant eye at different spatial frequencies. The wide variety of contrast filtering is seen in these results (Fig. 4). To obtain these results we used the means of the results of the normal eyes. Since there is a range of sensitivity for normal eyes, the lower limit of the $95 \%$ confidence limit of normal sensitivity is seen in Fig. 4 as a horizontal dashed line. Results falling above this line (e.g., patient 1), while being below the normal mean, are not outside the $95 \%$ confidence limits of normal vision and so should be viewed as suspicious rather than definitely abnormal.

The filtering properties of these two IOL types in situ fell into three categories: low pass, band reject, and band pass. In the low pass cases contrast was attenuated more at higher spatial frequencies. This is similar to optical blur. In the band reject cases contrast was selectively lost for only intermediate spatial frequencies. In the band pass case contrast was lost at low and high spatial frequencies but was relatively spared for intermediate spatial frequencies. 


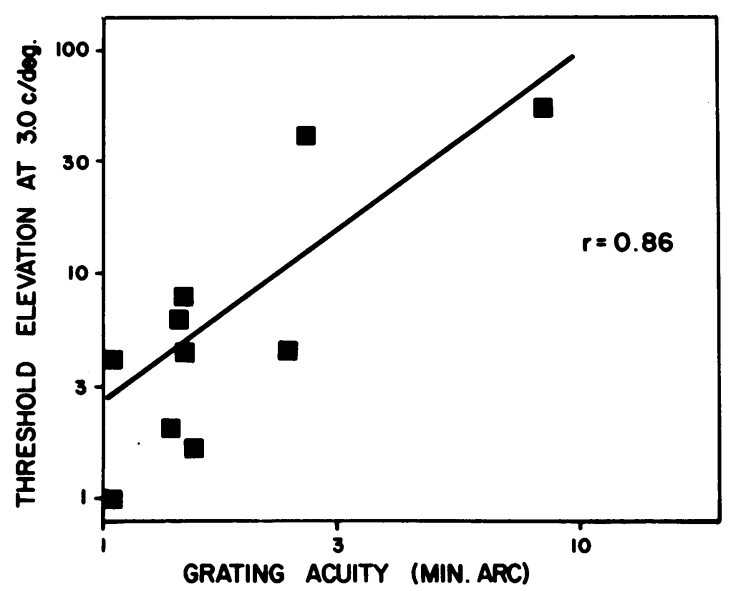

Fig. 3 Correlation between the contrast threshold loss at $3 \mathrm{c} / \mathrm{deg}$ and the grating acuity is plotted for the 10 eyes with intraocular implants. The non-zero y intercept indicates that grating acuity is not a very good predictor of contrast loss at intermediate spatial frequencies in some cases.

\section{Discussion}

The present clinical assessment of the optical performance of IOL implants in situ is accomplished by the measurement of visual acuity and reports by the patient. This is a very rudimentary assessment when one considers the accuracy with which the optical quality of isolated lenses can be specified with modulation transfer function (MTF) procedures. In this latter procedure contrast transmission can be accurately assessed for sine wave gratings of a wide range of spatial frequencies, and the exact form of the

Table 1 Visual acuities of 9 patients with IOLs

\begin{tabular}{llll}
\hline Patient & \multicolumn{2}{l}{ Visual acuities } & Type of $I O L$ \\
\cline { 2 - 3 } & $O D$ & $O S$ & \\
\hline 1 & $6 / 9$ & $6 / 7 \cdot 5$ & OD Fyodorov \\
2 & $6 / 9$ & $6 / 30$ & OS Fyodorov \\
3 & $6 / 7 \cdot 5$ & $6 / 6$ & OD Fyodorov \\
4 & $6 / 18$ & $6 / 18$ & OS Fyodorov \\
5 & $6 / 18$ & $6 / 18$ & OS Fyodorov \\
6 & $6 / 96$ & $6 / 15$ & OD Binkhorst \\
7 & $6 / 38$ & $6 / 7 \cdot 5$ & OD Binkhorst \\
8 & $6 / 9$ & $6 / 38$ & OU Fyodorov \\
9 & $6 / 6$ & $6 / 6$ & OS Fyodorov \\
\hline
\end{tabular}

Snellen visual acuities are listed for 10 patients with IOLs. The acuity chart was calibrated in log MAR (logarithmic minimum angular resolution) units of $0.1 \mathrm{log}$ intervals. According to this new and useful scheme the acuity of each line on the chart changes by a constant factor (for example, 1-26). We used this chart because of its greater accuracy, but we have converted these to Sncllen notation for case of comparison. Since the acuity step is finer than the usual clinical chart, our converted acuitics result in some unfamiliar Snellen fractions. contrast loss (that is, its filtering characteristics) can be obtained. Since the final court of appeal for IOLs is their optical performance in situ, we have adopted a psychophysical procedure analogous to the now well established MTF optical assessment procedure.

Our psychophysical procedure involves a comparison between the measurement of contrast thresholds for various spatial frequency sinewave gratings for normal eyes and those with implanted lenses. The ratio of these sensitivities across spatial frequency gives a psychophysical estimate of the modulation transfer properties of the implants and media environment itself. This procedure relies upon the assumption that any differences between normal eyes and eyes with lens implants are due to the implants and media environment. Aside from patient 6 , none of the patients showed any ophthalmologically detectable postsurgical complications.

Interestingly we found that the contrast loss produced by these two examples of iris clipped lenses was considerable and variable in form. In most cases the conventional acuity test would not have detected this (see Table 1), and unless the patient had one normal eye with which to compare it might not have been easily identified by the patient. For some patients contrast was lost only at high spatial frequencies (low pass). This is similar to blur and thus likely to be the only type of transmission loss detected and adequately assessed by acuity tests. For another group of patients contrast was lost selectively at mid spatial frequencies. This would not be detected at all by standard acuity tests. In the one case in which there were postsurgical complications (patient 6) contrast was lost at low and high spatial frequencies but relatively spared for intermediate spatial frequencies. The extent of the visual loss in this case would also have been grossly underestimated by acuity tests. The variety and extent of the contrast loss due to these intraocular implants (some loss is hidden and restricted to only mid spatial frequencies) argues strongly for assessment of visual performance of these patients to include more than just the limit of resolution (acuity tests).

As is the case in all visual loss due to optical anomalies, any threshold loss has similar consequences for suprathreshold contrast targets. For example, if the contrast threshold is raised by a factor of 10 due to optical degradation of an intraocular implant, then it is also true that any suprathreshold contrast will be similarly reduced. Thus a $100 \%$ contrast target will be reduced to $10 \%$. This means that the contrast loss at threshold reported here will have important consequences for visual performance at all, (including high) contrast levels. In order to estimate the possible perceptual consequences of the contrast losses observed with these implants a photo- 


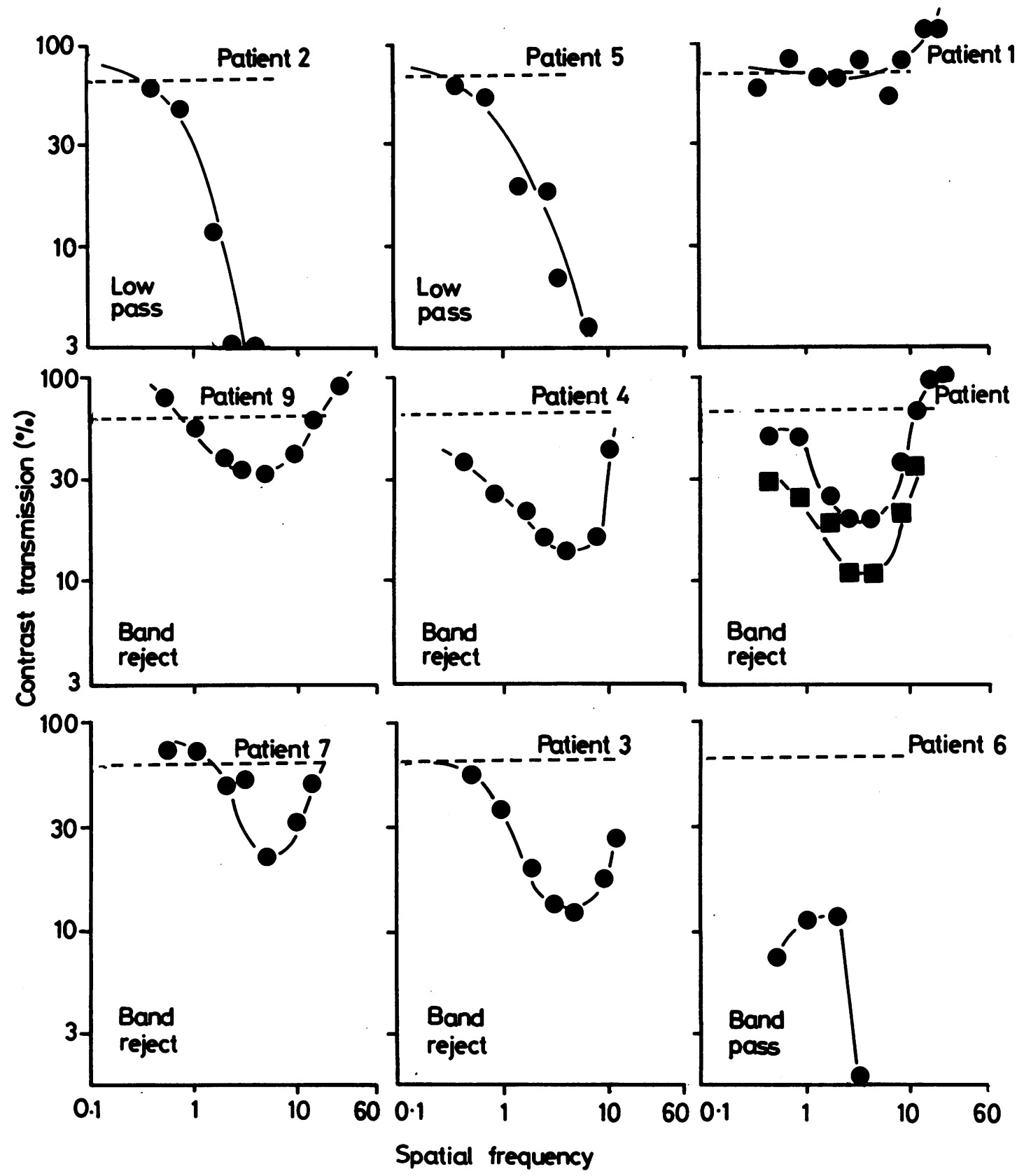

Fig. 4 The average contrast sensitivity results shown in Fig. 2 for the 10 normal eyes have been divided by the contrast sensitivity of the implant eye at each spatial frequency to obtain the contrast transmission characteristics (\% transmission) of the intraocular implant. The dash line indicates the lower boundary of the $95 \%$ confidence limit of the normal population. Results above the dash line are not significantly different (patient 1) from the normal population. The contrast transmission properties fall into three categories: low pass, band reject, and band pass (see text). 
Filtered scene

(a)

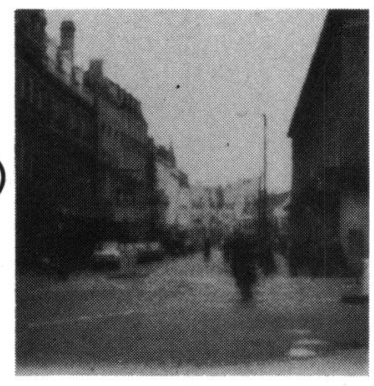

(b)

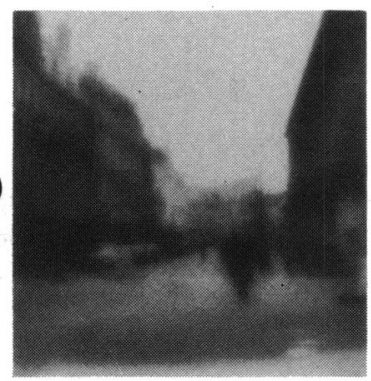

(C)
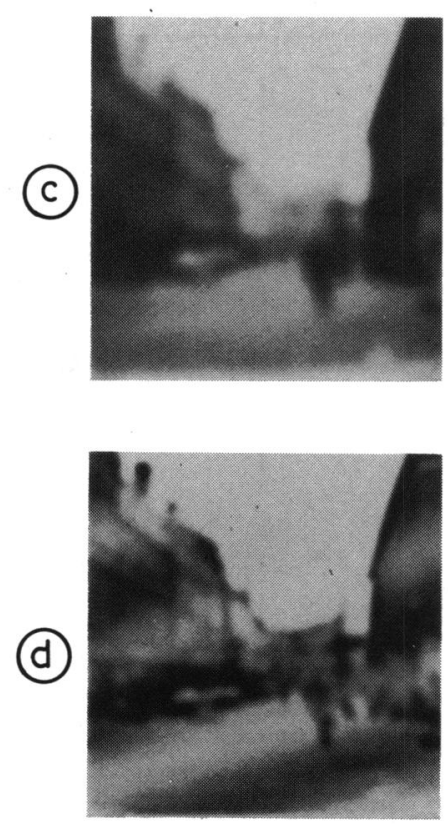

Contrast transmission
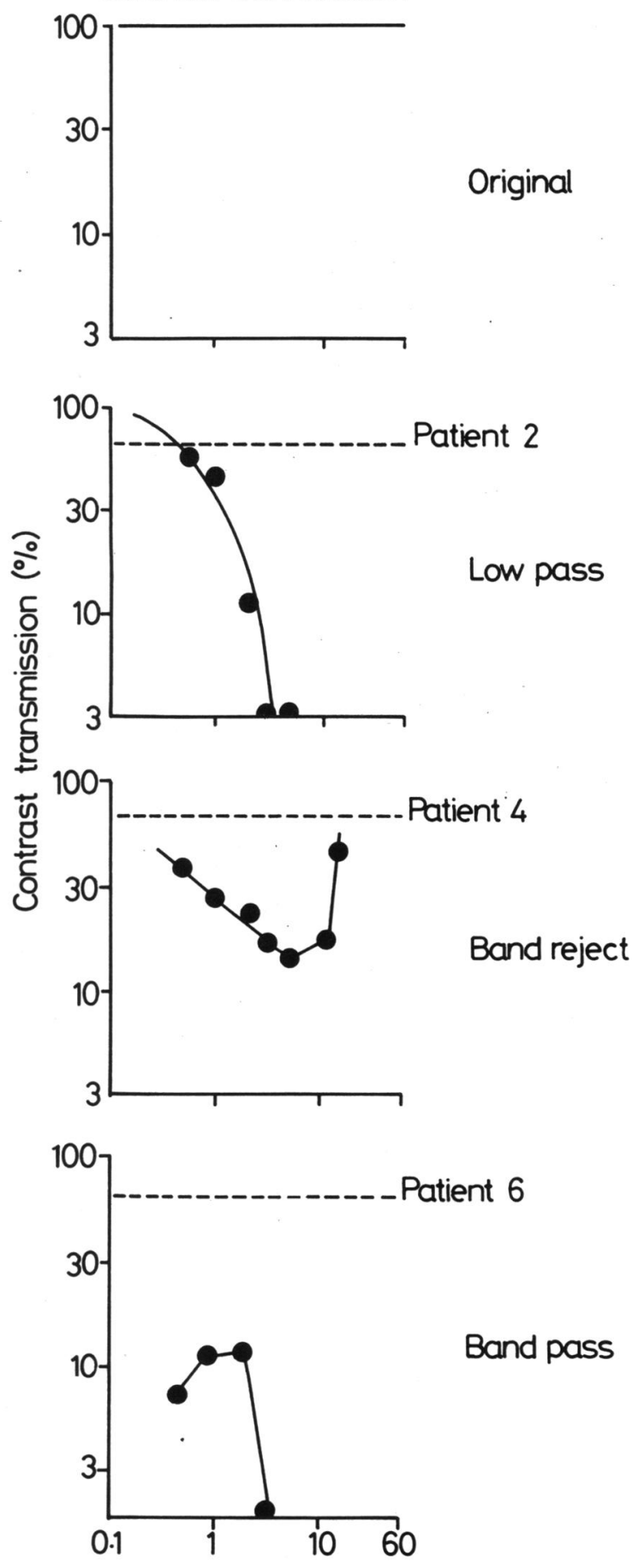

Spatial frequency (C/deg)

Original

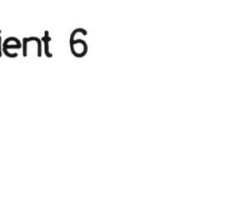

Band pass 
Fig. 5 Anoutdoor scene (A) is

digitally filtered in accordance with

the three types of contrast loss

shown to occur as a result of $I O L$

implant. Whereas the low pass (B)

and band reject $(\mathrm{C})$ properties result

from the IOL and media

environment, the band pass

properties (D) were contaminated

with post-surgical macular oedema.

These pictures give only a crude

indication of the perceptual

consequences of measured losses in

contrast.

graph of a visual scene was spatially filtered in accordance with the three different types of contrast loss reported here (Fig. 5). Fig. 5A was digitally sampled and reconstructed without any modification to show the quality of the filtering process itself. Fig. 5B, 5C, 5D represent contrast losses of spatial frequencies in accordance with the three types (low pass, band reject, and band pass) of transmission loss found in the present study.

Note that in all three cases there is a significant loss in optical quality. Patient 4 , whose transplant displayed band reject transmission properties, has the least severely affected acuity (Snellen 6/18; grating acuity $20 \mathrm{c} / \mathrm{deg}$ ). Since contrast is only lost for intermediate spatial frequencies much of the high frequency or fine detail is present in the filtered image, but it is difficult to discern objects of intermediate size. Patients 2 and 6 show very different transmission properties, but their grating acuities are identical ( $3 \mathrm{c} / \mathrm{deg})$. Their filtered images are noticeably different as are their Snellen acuities (patient 2, $6 / 30$; patient $6,6 / 96$ ). The patchy nature of patient 6 's filtered image is because of an added loss of contrast at low spatial frequencies. These pictures demon- strate the diversity of perceptual effects from the different filtering properties of these lenses.

\section{CONCLUSION}

In conclusion our results show that contrast loss can be severe, particularly when there are surgical complications. There can be many different types of contrast loss with IOLs, and these can be adequately assessed by our measuring thresholds at high, low, or intermediate spatial frequencies. Snellen acuity measurements are not adequate to detect these losses or to appreciate their perceptual consequences. We advise that contrast threshold measurements should be used to assess the effectiveness of these devices for low, medium, and high spatial frequencies.

This study is supported in part by the Natural Sciences and Enginecring Research Council of Canada (Grant No A3449), the Mcdical Rescarch Council of Great Britain, and the Wellcome Trust. We thank Mr I Piotrowski for producing the photographic plates. RFH is a Wellcome senior lecturer.

\section{References}

1 Ridley H. Intraocular acrylic Ienses. Trans Ophthalmol Soc UK 1951; 71: 617-21.

2 Nordlohne ME. The intraocular implant lens: development and results with special reference to the Binkhorst lens. 2nd ed. Baltimore: Williams and Wilkins, 1975.

3 Stark W. The FDA report on intraocular lenses. Ophthalmology 1983; 90: 311-7.

4 Kayes J. Recent experience in lens implantation. Presented at the Washington University Eye Alumni Mecting. St Louis. April 1981. Cited by Bobrow JG. Drews RC. in 'Iris clip lens implantation.' In: Langton RHS, ed. Intraocular lens implantation. International Ophthalmology Clinics. Boston: Littlc. Brown. 1981.

5 Hurite FG, Lempert SL. Iridocapsular intraocular lens and extracapsular cataract surgery. In Langton RHS. ed. Intraocular lens implantation. International Ophthalmology Clinics. Boston: Little, Brown, 1981.

6 Campbell FW. Green DG. Optical and retinal factors effecting visual resolution. J Physiol (Lond) 1965; 181: 576-93.

7 Hess R. Woo G. Vision through cataracts. In'est Ophthulmol Visual Sci 1978; 17: 428-35.

8 Woo G. Dalizel C. A pilot study of contrast sensitivity assessment of CAM treatment of amblyopia. Acta Ophthalmol $(K b h) 1981$ : 59: $35-7$. 